\title{
Imaging multiple phases of neurodegeneration: a novel approach to assessing cell death in vivo
}

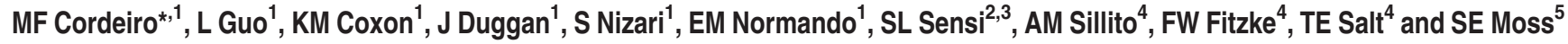

Nerve cell death is the key event in all neurodegenerative disorders, with apoptosis and necrosis being central to both acute and chronic degenerative processes. However, until now, it has not been possible to study these dynamically and in real time. In this study, we use spectrally distinct, well-recognised fluorescent cell death markers to enable the temporal resolution and quantification of the early and late phases of apoptosis and necrosis of single nerve cells in different disease models. The tracking of single-cell death profiles in the same living eye over hours, days, weeks and months is a significant advancement on currently available techniques. We identified a numerical preponderance of late-phase versus early-phase apoptotic cells in chronic models, reinforcing the commonalities between cellular mechanisms in different disease models. We showed that MK801 effectively inhibited both apoptosis and necrosis, but our findings support the use of our technique to investigate more specific anti-apoptotic and anti-necrotic strategies with well-defined targets, with potentially greater clinical application. The optical properties of the eye provide compelling opportunities for the quantitative monitoring of disease mechanisms and dynamics in experimental neurodegeneration. Our findings also help to directly observe retinal nerve cell death in patients as an adjunct to refining diagnosis, tracking disease status and assessing therapeutic intervention.

Cell Death and Disease (2010) 1, e3; doi:10.1038/cddis.2009.3; published online 14 January 2010

Subject Category: Neuroscience

This is an open-access article distributed under the terms of the Creative Commons Attribution License, which permits distribution and reproduction in any medium, provided the original author and source are credited. This License does not permit commercial exploitation without specific permission.

Cell death and neuronal loss are the key pathological drivers of neurodegeneration in conditions such as Alzheimer's disease (AD), Parkinson's disease, Huntington's disease and glaucoma, with increasingly recognised common elements and triggers (e.g., oxidative stress, mitochondrial dysfunction, excitotoxicity and misfolded protein aggregation) and a convergence on downstream events. ${ }^{1,2}$ For example, the protein $\beta$-amyloid $(\mathrm{A} \beta)$ seems to be involved in the eye in a similar way as it is in the brain, with retinal $A \beta$ deposition being strongly implicated in both $A D$ and glaucoma. ${ }^{3-6}$

Live cell imaging has been widely used to investigate neuronal dysfunction in cultured cells in vitro, which, together with fluorescent multiple labelling, permits visualisation of different cell activities and distinct molecular localisation patterns. The use of annexin $\mathrm{V}$ and propidium iodide (PI) as cell death markers has become an established method for differentiating between the two major forms of cell death namely, apoptosis and necrosis. However, until now, inves- tigation of the progression and dynamics of the distinct phases of neurodegenerative disease at the cellular level has depended on histological or in vitro analyses. ${ }^{7}$

Imaging the different phases of nerve cell death in vivo would significantly advance our ability to understand the disease and its natural history, with direct applications to the patient. It would allow an investigation into the time course of events in relation to different modulators, and also provide insight into the spatial patterns of cell death over periods of days, weeks or longer. Furthermore, given the emergence of mechanistic commonalities between different neurodegenerative diseases, such as $\mathrm{AD}$ and glaucoma, ${ }^{5,6}$ an appreciation of the spatio-temporal dynamics of cell death in one disease could enhance our understanding of other diseases, in terms of both the molecular pathophysiology and the potential therapeutic avenues.

Compared with other mammalian organs, the eye offers unique opportunities for imaging because the clear optical

\footnotetext{
${ }^{1}$ Glaucoma and Retinal Neurodegeneration Research Group, UCL Institute of Ophthalmology, University College London, Bath Street, London EC1V 9EL, UK; ${ }^{2}$ Molecular Neurology Unit, Center of Excellence on Aging (CeSi), University 'G. d'Annunzio', Chieti Scalo 66013, Italy; ' ${ }^{3}$ Department of Neurology, University of California-Irvine, Irvine, CA 92697-4292, USA; ${ }^{4}$ Visual Neurosciences, UCL Institute of Ophthalmology, University College London, Bath Street, London EC1V 9EL, UK and ${ }^{5}$ Cell Biology Divisions, UCL Institute of Ophthalmology, University College London, Bath Street, London EC1V 9EL, UK

${ }^{*}$ Corresponding author: MF Cordeiro, Glaucoma and Retinal Neurodegeneration Research Group, UCL Institute of Ophthalmology, University College London, Bath Street, London EC1V 9EL, UK. Tel: + 44207608 6938; Fax: + 207608 6939; E-mail: M.Cordeiro@ucl.ac.uk

Keywords: neurodegeneration; retinal imaging; apoptosis; necrosis; Alzheimer's disease; glaucoma

Abbreviations: AD, Alzheimer's disease; A $\beta, \beta$-amyloid; PI, propidium iodide; Dil, 1,1'-dioctadecyl-3,3,3',3'-tetramethylindocarbocyanine perchlorate; cSLO, confocal scanning laser ophthalmoscope; DiAsp, (4-(4-(didecylamino)styryl)-N-methylpyridinium iodide; SSP, staurosporine; RGC, retinal ganglion cell; PMA, phorbol myristate acetate; OHT, ocular hypertension; IOP, intraocular pressure; NMDA, $N$-methyl-D-aspartic acid

Received 23.9.09; revised 02.10.09; accepted 02.10.09; Edited by G Melino
} 
media allow a direct visualisation of disease processes as they occur. Recently, we described a technique for real-time imaging of retinal ganglion cell (RGC) apoptosis in the mammalian eye using confocal scanning laser ophthalmoscopy and fluorescent-labelled annexin $\mathrm{V}^{1}{ }^{1}$ In this study, we used $\mathrm{PI}$ and 1,1'-dioctadecyl-3,3,3',3'-tetramethylindocarbocyanine perchlorate (Dil) as additional cellular markers to distinguish between early and late apoptotic and necrotic phases of single nerve cell death in vivo. We carried this out in established acute, chronic and treated models of neurodegeneration ${ }^{1,6,8}$ to highlight the capabilities of snapshot and longitudinal assessment. Changes in individual neurons were visualised in the retina over hours, days and weeks, as were the effects of modulating and neuroprotective agents, and levels of cell death phase quantified.

In conclusion, our new technique shows the retina to be an ideal experimental model that provides compelling opportunities for the quantitative monitoring of disease mechanisms and dynamics in experimental neurodegeneration. It also helps to directly observe retinal nerve cell death in patients as an adjunct to refining diagnosis, tracking disease status and assessing therapeutic intervention. ${ }^{6,9}$

\section{Results}

Customising imaging equipment enables fluorescent triple-labelling detection. To simultaneously detect three different fluorochromes, we first customised a prototype Zeiss (Oberkochen, Germany) confocal scanning laser ophthalmoscope (cSLO) ${ }^{1}$ to enable the detection of different emission wavelengths. The parameters used were based on well-established fluorescently tagged cell markers, selected because of their distinct spectral properties and minimal overlap, and the emitted light collection was optimised with appropriate filters (Figure 1). For identification of apoptotic cells, we used Alexa Fluor 488labelled annexin V (Invitrogen, Paisley, UK), which has an excitation and emission maxima of 495 and $519 \mathrm{~nm}$, respectively, with an argon laser and a solid-state photodetector with a $521 \mathrm{~nm}$ cut-off filter and a $550 \mathrm{~nm}$ cut-on filter. ${ }^{1}$ To identify necrotic and late-apoptotic RGCs, we used PI with an excitation and emission maxima of 532 and $649 \mathrm{~nm}$, respectively, with detection using a diode-pumped crystal green laser with a wavelength of $532 \mathrm{~nm}$ and a $650 \mathrm{~nm}$ cut-off filter on the photodetector. After an empirical assessment

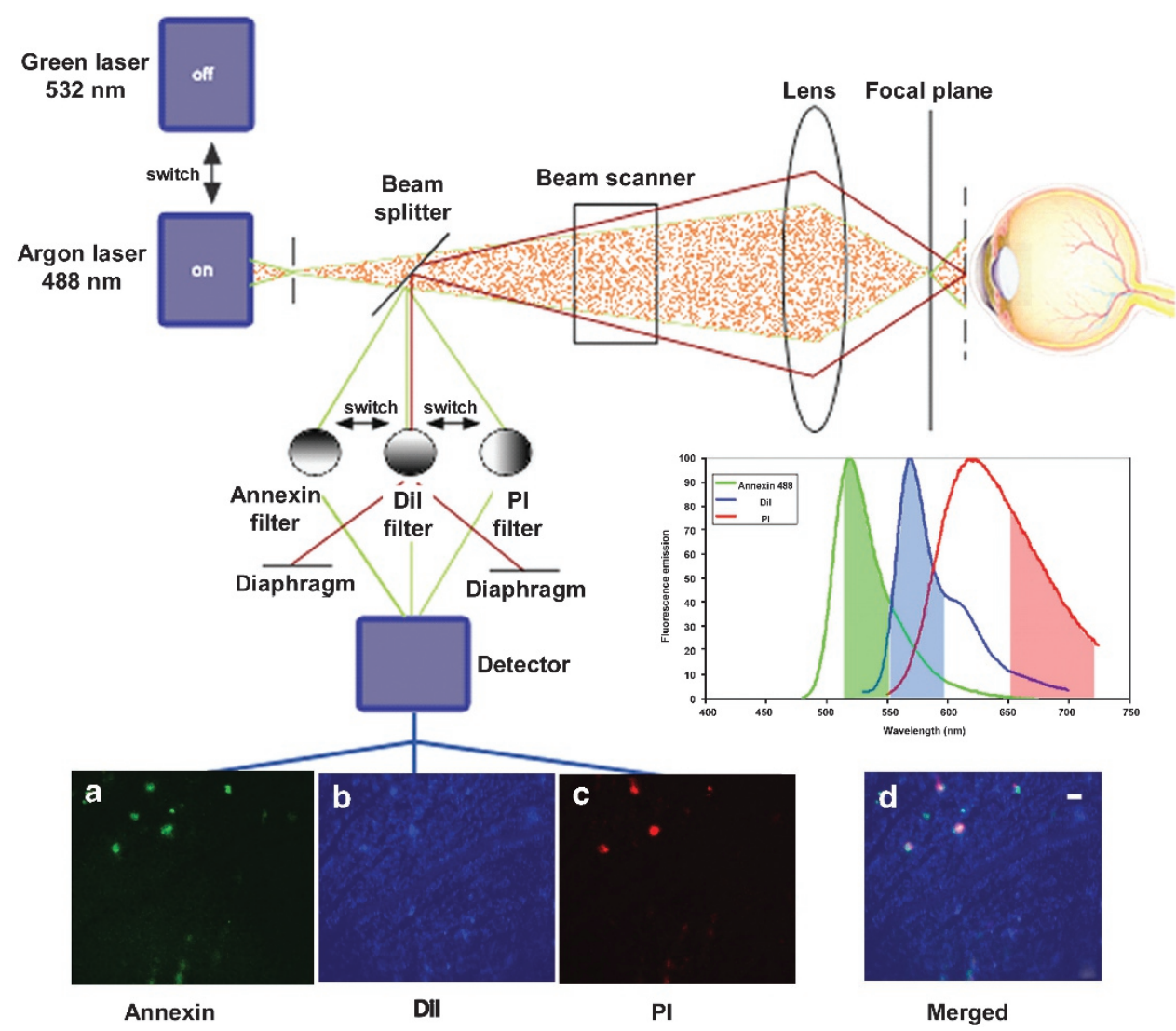

Figure 1 Confocal scanning laser ophthalmoscopy (CSLO) and detection of fluorescent-labelled cells. Simultaneous detection of multiple markers was achieved by customising the cSLO as follows: for identification of annexin 488, an argon laser wavelength of $488 \mathrm{~nm}$ and a solid-state photodetector with a $521 \mathrm{~nm}$ cut-off filter and a $550 \mathrm{~nm}$ cut-on filter were used; for Dil, a diode-pumped crystal green laser with a wavelength of $532 \mathrm{~nm}$ was used with $550 \mathrm{~nm}$ cut-off and $600 \mathrm{~nm}$ cut-on filters; the same laser was used for PI visualisation but with a $650 \mathrm{~nm}$ cut-off. The inset graph shows the emission spectra of cellular marker fluorochromes used in this study. Alexa Fluor 488labelled annexin V (green; annexin 488), Dil (blue; 1, 1' -dioctadecyl-3,3,3',3'-tetramethylindocarbocyanine perchlorate) and propidium iodide (red; PI) have excitation/emission maxima of 495/519,550/570 and 532/649 nm, respectively. (a-d) Monochrome images from each retinal area were acquired using the appropriate laser setup and colourised. Three-colour images were produced by combining each of the grey scale images from the cLSO into the green (a, annexin 488), blue (b, Dil) and red channels (c, PI) of an RGB colour image, and merged to form the image shown in (d) (scale bar $100 \mu \mathrm{m})$ 
of several different compounds, including DiAsp (4-(4(didecylamino)styryl)- $N$-methylpyridinium iodide (4-Di-10-Asp)), Fluorogold and Rhodamine B, we determined that Dil was the most suitable RGC marker in our rat models, with an excitation and emission maxima of 550 and $570 \mathrm{~nm}$, respectively. The same laser used for PI was used for Dil visualisation, but with $550 \mathrm{~nm}$ cut-off and $600 \mathrm{~nm}$ cut-on filters.

This system permitted multi-wavelength imaging with high resolution and sensitivity, and minimal bleed-through (Figure 1). Filters were specifically chosen for the fluorochromes used, but in principle, a diverse number of fluorescent markers used in a wide range of established cell biology protocols could be studied with appropriate modification to the cSLO system - highlighting the use of the retina as a model for studying in vivo cellular processes.

In vivo imaging allows longitudinal tracking of changes in cell markers over time. Having defined the functional parameters of the technology, we next investigated its application in vivo using animal models of neurodegeneration. Staurosporine (SSP), a well-known non-selective protein kinase inhibitor, is routinely used as a potent inducer of neuronal apoptosis in in vitro assays. ${ }^{10}$ We have previously established that SSP may be conveniently used in vivo to induce a rapid and fairly synchronous wave of RGC apoptosis that is readily detected using annexin $V^{1,11}$ Therefore, we first studied the effects of SSP in rats in which
RGCs had been retrogradely labelled with Dil, followed by intravitreal delivery of SSP, annexin V-488 and PI.

In this model, time-lapse video sequences (Figures $2 \mathrm{a}-\mathrm{e}$ and Supplementary Videos 1 and 2) of single retinal areas over $5 \mathrm{~h}$ revealed a random distribution and appearance of apoptotic and necrotic cells, which was particularly highlighted by the 3D surface plot (Supplementary Video 2), and the average intensity projections of the time-lapse sequence shown in Figures $2 f$ and $g$ (generated by ImageJ RGB Zprojector plugin). A graphical analysis of the image sequence histograms demonstrated that the average intensity profiles of annexin $\mathrm{V}$ and $\mathrm{PI}$ changed significantly over time (examples shown in Figure $2 \mathrm{~h}$ ).

These results demonstrate proof of principle that this technique enables the tracking of changes in individual retinal cells in the same eye over time, with the ability to temporally and quantitatively analyse changes in fluorescence intensity. To our knowledge, this is the first demonstration of the use of high-resolution multi-wavelength imaging in visualising the death of single neurons in vivo.

Identifying patterns of tracked single-cell death markers over time in vivo. We next assessed whether the multilabelling of cells in vivo allowed the resolution of different patterns of apoptotic and necrotic cell death. Owing to the rapid kinetics and predominantly apoptotic mode of cell death induced by SSP, we again investigated simultaneous triple labelling in rats. Although the precise kinetics of the onset of
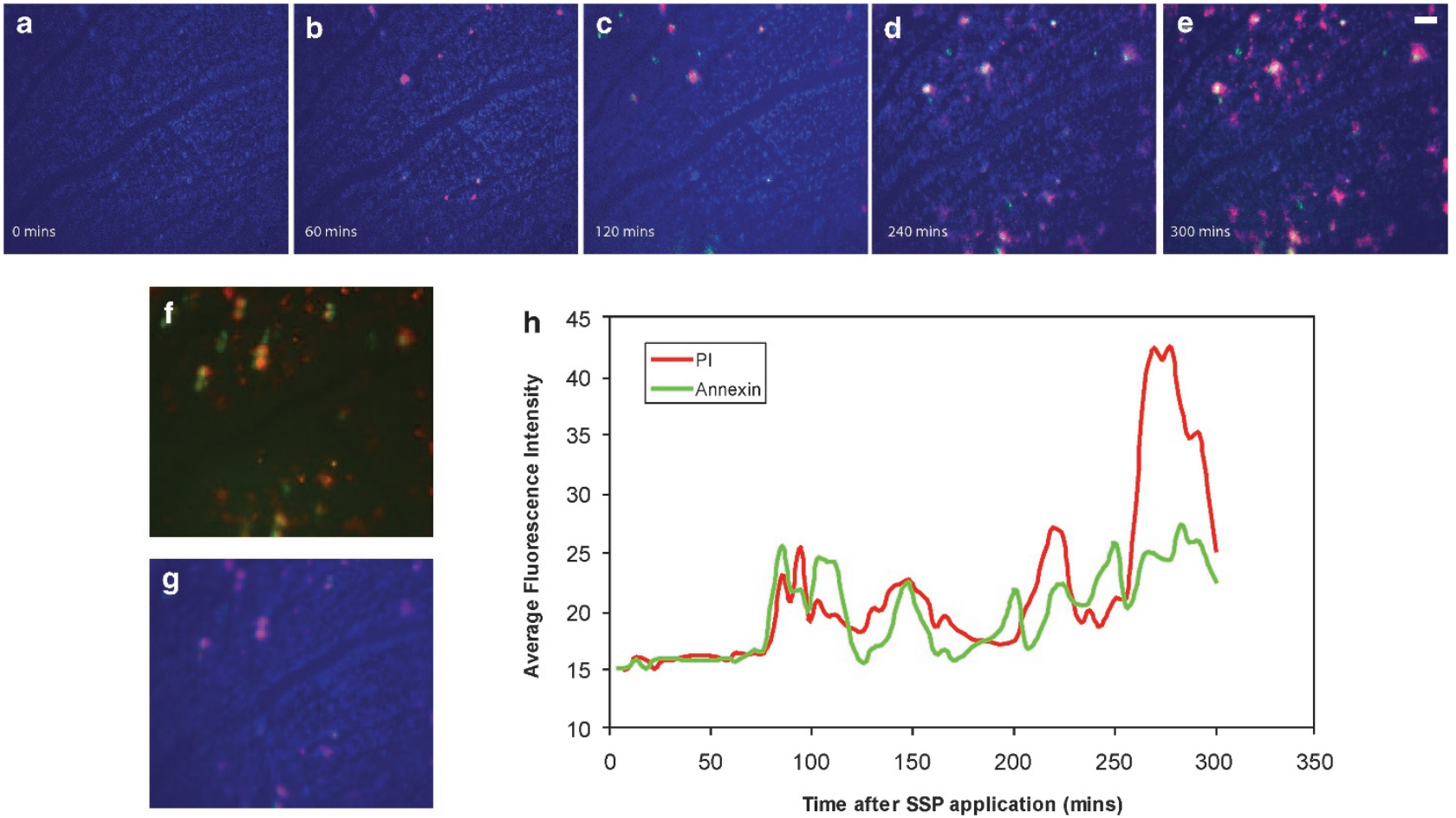

Figure 2 Time-lapse video analysis showing changes in cell markers in vivo. (a-g) Stills taken from video sequence (Supplementary Video 1) in an SSP-treated rat eye with labelled RGCs (Dil, blue) and intravitreal annexin V (green) and propidium iodide (PI, red) reveal a random distribution and appearance of annexin V-and PI-stained cells, but demonstrate the clear increase in PI staining over time. (DI, blue; annexin V, green; propidium iodide, red; time points and scale bar $100 \mu \mathrm{m}$ as indicated). (f, $\mathbf{g}$ ) Average fluorescence intensity projections of video sequences using ImageJ RGB Zprojector plugin with annexin/PI (f) and all three channels $(\mathbf{g})$. (h) Temporal analysis of the average annexin (green) and PI (red) intensities taken from each image sequence histogram demonstrates that the profiles of annexin and PI change significantly over time, with a marked increase in late PI staining 

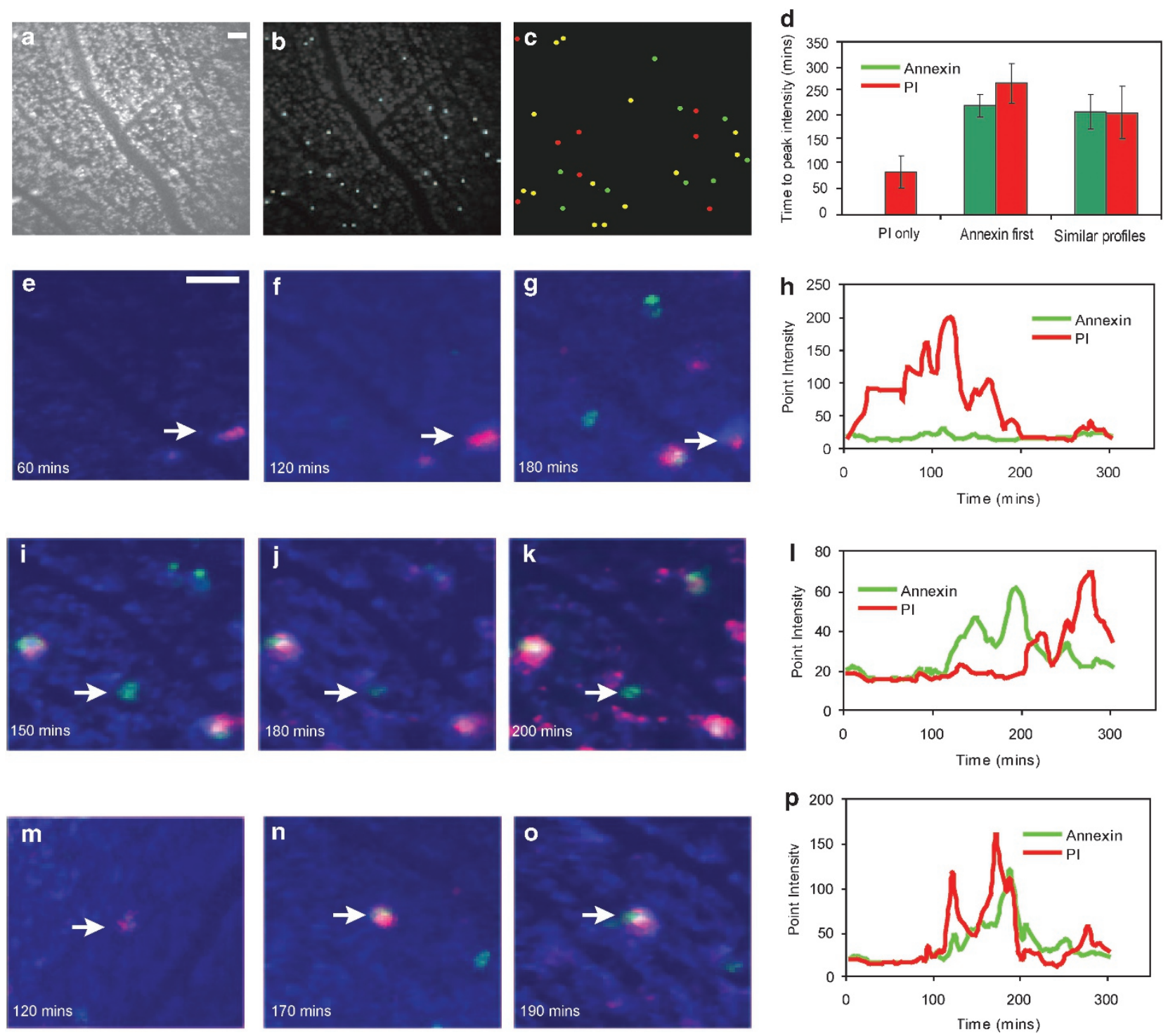

Figure 3 Identification of different patterns and phases of cell death in vivo. (a, $\mathbf{b})$ Random sample $(n=30)$ of single cells selected according to whether they were Dil positive (white spots, Dil retrogradely labelled retinal ganglion cells, a) and stained with annexin V-488, PI or both during time-lapse video (light blue spots, b). (c-p) Three patterns of fluorescent labelling were identified: 'PI only' (red spots, (c), and the representative cell, arrowed in (e-g) video stills with the corresponding signal intensity profile in (h)), 'Annexin first' (annexin-V positive and subsequently PI positive, green spots (c, i-l)), 'similar profiles' (positive labelling for both dyes at the same time, yellow spots, (c, $\mathbf{m}-\mathbf{p})$ ). The mean times to peak intensity (d) revealed that the appearance of the 'PI only' cells was significantly quicker than either the 'annexin first' or the 'similar profiles' cells. Intensity profiles of annexin V and PI changed significantly over time (h, I, p). These results provide evidence that neuronal cells in vivo show considerable heterogeneity with regard to the kinetics of necrotic and apoptotic cell death, and also the relative timing of phosphatidylserine exposure (early phase) and nuclear condensation (late phase). Time points and scale bar $100 \mu \mathrm{m}$ as indicated

annexin- $\mathrm{V}$ positivity and the development of $\mathrm{PI}$ staining varied from cell to cell, single-cell tracking allowed the delineation of the natural history of the fluorescence patterns.

In this SSP model, ${ }^{1,9}$ single cells were identified from stills, and a random sample $(n=30)$ was selected based on whether they were Dil positive (i.e., white spots identifying single RGCs, Figure 3a) and stained with annexin V-488, PI or both. A video analysis of selected tracked individual cells fulfilling these criteria (artificially labelled as light blue spots in Figure 3b) using ImageJ software and the Time Series Analyzer plugin allowed us to construct the intensity profiles of annexin $\mathrm{V}$ and PI. From these profiles, it was found that typically three patterns of fluorescent labelling were observed, as illustrated in Figure 3c, which is a colour map showing the distribution of cells and their staining patterns. First, some cells were found to be labelled only with PI ('PI only', red spots, Figure $3 \mathrm{c}$ ), others were initially annexin $\mathrm{V}$ positive and subsequently became PI positive ('Annexin first', green spots, Figure 3c), whereas others became positive for both dyes at approximately the same time ('similar profiles', yellow spots, Figure 3c). The patterns of fluorescent labelling seemed to be randomly distributed.

The tracking of annexin V-488 and PI staining in single cells is clearly shown in the magnified video stills illustrating representative cells displaying specific patterns of fluorescent labelling (arrows in Figures $3 \mathrm{e}-\mathrm{g}$ indicating ' $\mathrm{PI}$ only'; i-k 
'Annexin first'; and m-o 'similar profiles'), with the corresponding graphs of signal intensity over time (Figure $3 \mathrm{~h}, \mathrm{I}$ and $\mathrm{p}$ ). In each illustrative case, the intensity profiles of annexin $\mathrm{V}$ and $\mathrm{PI}$ changed significantly over time. The mean timings to peak intensity of this random sample (Figure $3 \mathrm{~d}$ ) revealed that ' $\mathrm{PI}$ only' cells tended to show significantly shorter kinetics than either the 'Annexin first' or the 'similar profiles' cells. In addition, cells that became annexin $\mathrm{V}$ positive invariably also became PI positive, either within an hour or coincidentally.

These results provide evidence that neuronal cells in vivo show considerable heterogeneity with regard to the kinetics of necrotic and apoptotic cell death marker staining, and also, by inference, the relative timing of phosphatidylserine exposure (early-phase apoptosis) and nuclear condensation (latephase apoptosis).

Assessing the phases of cell death in vivo. In vitro FACS analysis enables cells to be classified as being in early apoptosis (annexin V positive only), necrosis (PI only) or late-phase apoptosis (both annexin $\mathrm{V}$ and $\mathrm{PI}$ positive). To quantify the magnitude of late-phase apoptosis in vivo, we studied individual levels of annexin V, PI and their colocalisation using our recently developed $A \beta$ model of RGC death. $^{6}$ The advantages of this methodology are well established in the field of cell biology, and provide a snapshot of the status of RGC death at any one time. We also evaluated the effects of treatment with the $N$-methyl-Daspartic acid (NMDA) channel blocker MK801, using the same analytical methods.

Using the $\mathrm{A} \beta$ model, we assessed the effects of intravitreal $\mathrm{A} \beta\left(\mathrm{A} \beta_{25-35}\right)$, which was recently shown by us to induce $\mathrm{RGC}$ apoptosis in rodent eyes. ${ }^{6}$ Significant RGC death occurred $72 \mathrm{~h}$ after intravitreal administration of $3.40 \mathrm{nmol} A \beta_{25-35}$ to the right eye, a time that, in previous studies, was shown by us to yield peak levels of apoptosis (Figure 4). The left eye of each animal was treated with intravitreal $0.40 \mathrm{nmol}$ MK801 at the same time as $\mathrm{A} \beta$. Wide-angle retinal images were recorded $2 \mathrm{~h}$ after intravitreal annexin (green) and $\mathrm{PI}$ (red) staining, as previously described. A typical image of an $\mathrm{A} \beta_{25-35}$-treated right eye (Figure $4 \mathrm{a}$ ) compared with the left MK801-treated eye of the same mouse (Figure 4b) demonstrates that MK801 treatment reduces the numbers of annexin $\mathrm{V}$ (green)- and PI (red)-positive cells.

To assess the relative levels of early apoptosis, late apoptosis and necrosis, we studied the co-localisation of $\mathrm{PI}$ and annexin $\mathrm{V}$ using ImageJ software with the Intensity Correlation Analysis plugin. ${ }^{12}$ Colour and raw scatter plots of annexin and PI fluorescence intensity were constructed from plugin-generated data for each animal. The corresponding scatter plots (blue) from the eyes shown in Figures $4 a$ and $b$ are illustrated in Figures $4 \mathrm{c}$ and $\mathrm{d}$. Data were analysed in a manner similar to that of data derived by in vitro FACS analysis, with four quadrants (midlines shaded in colour overlay as red, green, blue and yellow) enabling separation of the different phases of cell death as annexin $\mathrm{V}$ positive (early apoptosis, green), annexin V and PI double positive (late apoptosis, yellow) and PI positive (necrosis, red). Thresholds were set automatically by the plugin for the analysis of each eye. We then used fluorescence intensity counts from the raw scatter plots to quantify each phase of cell death. MK801 treatment clearly reduced the overall levels of cell death (total), but more particularly, the number of cells in late-phase apoptosis and necrosis (Figure 4e). Interestingly, there was a small increase in the number of early-phase apoptotic cells $(\mathrm{Anx}+)$ in MK801-treated eyes (Figure 4e). As these are destined to become $\mathrm{Anx}+/ \mathrm{PI}+$, this suggests that, although the results generally support the neuroprotective role of NMDA antagonists in $\mathrm{A} \beta$-induced neuronal cell death, ${ }^{13}$ it may be that the protective effects of MK801 seem transient because of its single application in these experiments.

To further assess the analytical power of the technique, we next investigated whether the level of oxidative stress in $\mathrm{A} \beta$ treated eyes influenced the phase of cell death and whether this was possible to be visualised in vivo. Live cell imaging of glial/neuronal cultures has suggested that $A \beta$ increases neuronal oxidative stress by activating NADPH oxidase in microglia, an effect that is potentiated by phorbol myristate acetate (PMA). ${ }^{14}$ Figures $4 \mathrm{f}$ and $\mathrm{g}$ are taken from the right untreated $(\mathrm{f})$ and left $P M A$-treated $(\mathrm{g})$ eye of an animal treated $72 \mathrm{~h}$ previously with $3.40 \mathrm{nmol} \mathrm{A} \beta_{25-35}$. PMA $(0.016 \mathrm{nmol})$ or vehicle was administered at the same time as intravitreal annexin $\mathrm{V}$ and $\mathrm{PI}$, with imaging performed $2 \mathrm{~h}$ later. PMA increased the numbers of annexin $V$ (green)-positive cells (Figures $4 \mathrm{f}$ and $\mathrm{g}$ ), which was confirmed by the co-localisation scatter plots (Figures $4 \mathrm{~h}$ and i). Quantification of fluorescence in all animals revealed that PMA significantly $(P<0.05)$ increased the overall levels of cell death in the $A \beta$ model, in particular, the number of cells undergoing early-phase apoptosis (Figure 4j). These results support the hypothesis that PMA potentiates $\mathrm{A} \beta$-induced ROS damage via predominantly apoptotic pathways. ${ }^{14}$

Using the same methodology, we next compared the relative levels of cell death phases between the SSP and A $\beta$ acute models of RGC death, at the time points at which we previously showed peak RGC annexin V positivity to occur, that is, at 2 and $72 \mathrm{~h}$, respectively. ${ }^{1,6}$ Images were reanalysed for all treated animals using co-localisation scatter plots and the same methodology as described above. Figure $4 \mathrm{k}$ illustrates that both SSP and $\mathrm{A} \beta$ models exhibit similar patterns of cell death at the time of peak apoptosis. The cell death profiles in both models showed significantly more cells in the late phase of apoptosis $(P<0.05)$ as opposed to necrosis or early apoptosis.

The natural history of cell death in chronic glaucomarelated models. Having demonstrated the application of this technique in two acute models of RGC death, we next investigated models of chronic retinal neurodegeneration, which more closely resemble human disease. Our group has previously characterised, using in vivo and histological profiling of RGC apoptosis, an experimental glaucoma rat model of ocular hypertension (OHT) caused by chronically elevated intraocular pressure (IOP). ${ }^{1,9}$ RGCs were retrogradely labelled using previously described methods with $\mathrm{Dil}^{1,6}$ and animals were imaged repeatedly after administration of intravitreal annexin $\mathrm{V}$ at different time points to generate time-lapse video sequences (Figures $5 a-f$ and Supplementary Video 3). Assessment of intact labelled RGCs in the glaucoma model (identified by Dil, blue, Figure $5 \mathrm{a}$ ) revealed dramatic changes in single RGCs. As 
expected, when compared with the number of cells identified at baseline (Figure $5 b$ ) and at 3 weeks (Figure $5 c$ ), the number of labelled RGCs evident at 8 weeks was markedly reduced (Figure $5 \mathrm{~d}$ ) after surgical induction of elevated IOP. Analysis of apoptosis induction in these retinas revealed that those RGCs that were annexin $\mathrm{V}$ positive at
3 weeks (Figure 5e) disappeared by 8 weeks (Figure 5f), suggesting that a wave of apoptosis follows the initial IOP elevation insult, which is followed in turn by the clearance of dead cells.

We next analysed the time sequence of annexin $\mathrm{V}$ and Dil changes during this period. Using a method similar to that a
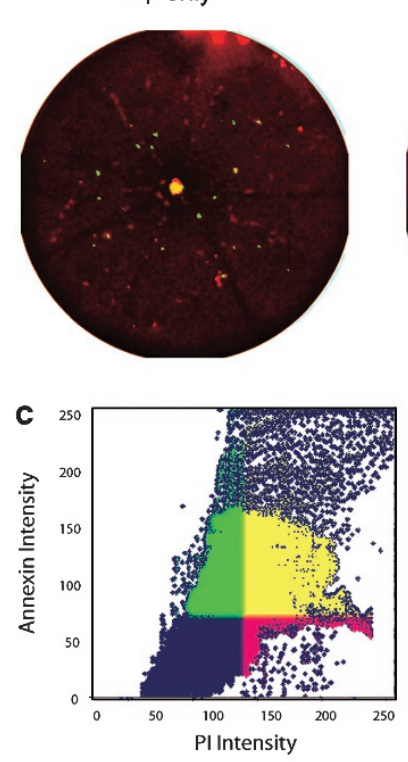

e 25000
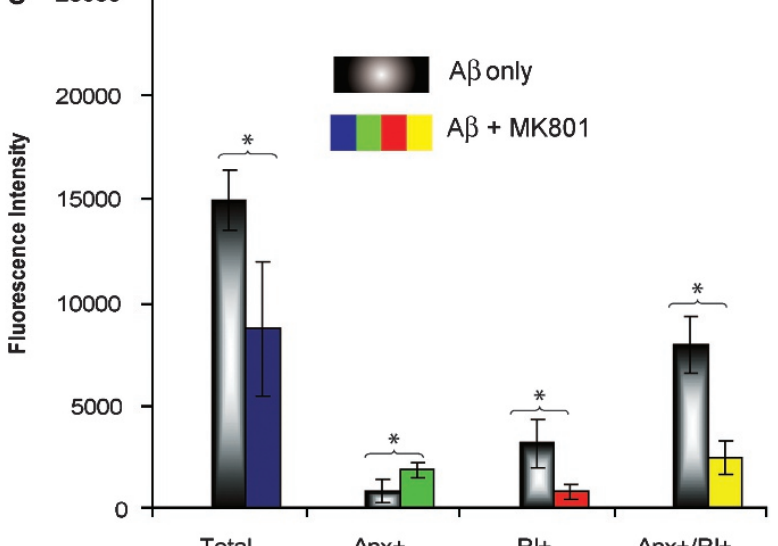

k b $\quad A \beta+M K 801$
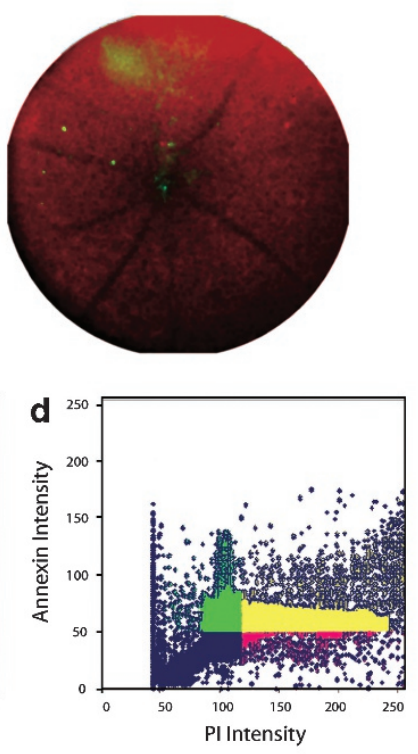

$A \beta$ only

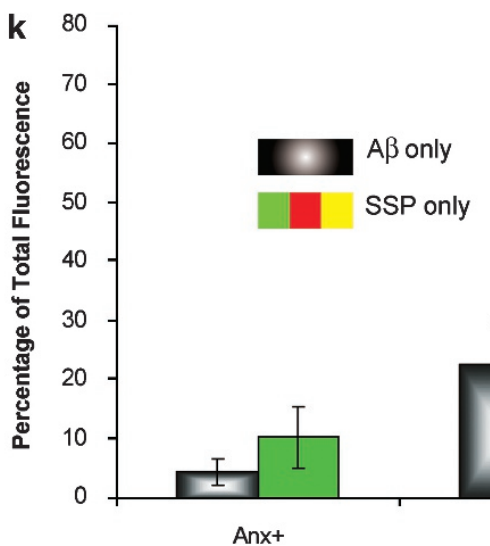

Anx+

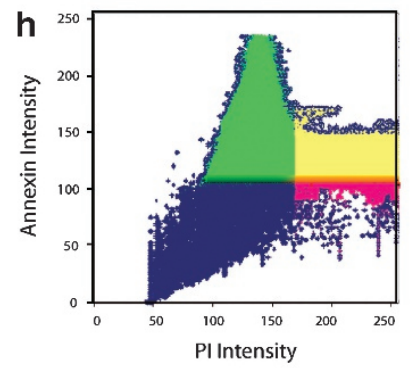

g $\quad A \beta+P M A$

f $\quad A \beta$ only

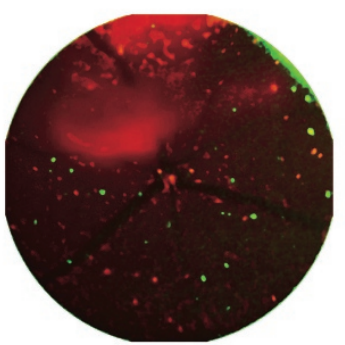

j

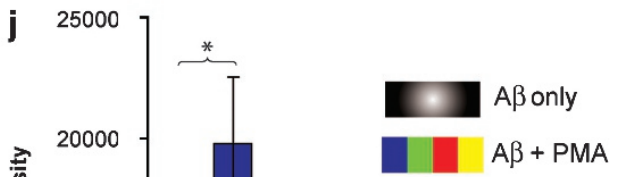

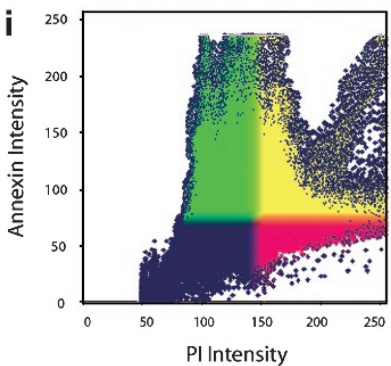

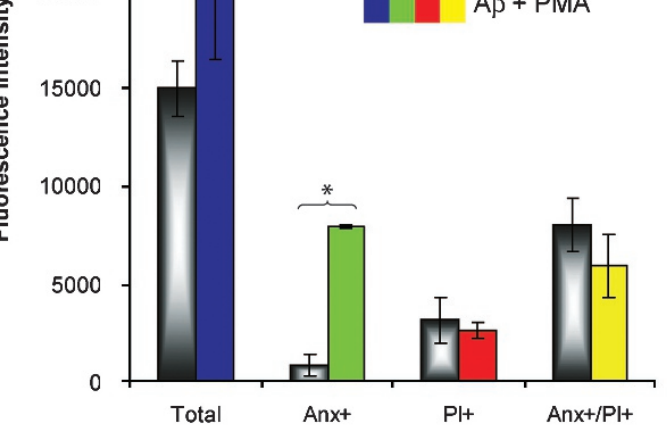


described earlier for Figure 2, we constructed a maximum intensity 3D projection of the time-lapse video sequences (Supplementary Video 3), using the Image J RGB Zprojector and JaCoP plugin. The projection enabled the quantitative assessment of maximal annexin $\mathrm{V}$ and Dil staining at each pixel in the same retinal area studied over time. Although there was a high overlap ratio of annexin $\mathrm{V}$ staining with Dil $(0.63$, confirming the presence of apoptosis at the level of the RGCs), there was a very low ratio (0.072) of Dil cells also positive for annexin $\mathrm{V}$. This demonstrates either that only some RGCs were ever annexin $\mathrm{V}$ positive and that apoptosis is not the only pathway responsible for cell death in this model, or that annexin V positivity is transient (as shown in Figure 3).

We then investigated the presence of apoptotic and necrotic cells using $\mathrm{PI}$ and annexin $\mathrm{V}$. At 3 weeks, in the OHT model, only a small proportion of RGCs was identified in early apoptosis compared with late apoptosis, as seen in a typical image in Figure 5g. Quantification of cell death phases was performed using the co-localisation scatter plots described previously. There was a significant $(P<0.05)$ increase in cells in late-phase apoptosis compared with either necrosis or early-phase apoptosis (Figure 5i). Indeed, most labelled cells were in late-phase apoptosis $(\mathrm{Anx}+/ \mathrm{PI}+)$, with relatively few cells exhibiting the diagnostic markers of necrosis $(\mathrm{PI}+)$ or early-phase apoptosis $(\mathrm{Anx}+)$. This distribution of dying cells is broadly similar to that seen in both the SSP and A $\beta$ models (Figure $4 \mathrm{k}$ ) at the time of peak apoptosis.

We next assessed whether neuroprotective treatment altered the relative numbers of cells in each of the cell death phases, using the NMDA antagonist MK801, which we have previously shown to be effective in this model. ${ }^{9}$ Treatment with MK801 at the time of IOP elevation significantly reduced the numbers of both annexin $\mathrm{V}$-and $\mathrm{PI}$-positive cells (Figure 5h). Co-localisation scatter plot analysis showed a significant reduction in total cell death markers $(P<0.05)$, and specifically a significant reduction in both late-phase apoptosis and necrosis, compared with untreated OHT eyes at 3 weeks after elevated IOP induction.

Assessing phases of cell death in the Alzheimer triple transgenic model. There is growing evidence of retinal involvement in $A D$, with the demonstration using histological techniques that RGC apoptosis occurs in double transgenic AD models. ${ }^{3,4}$ To determine whether phasing of RGC death could be assessed in vivo in another model of chronic neurodegeneration, we next applied our technique to a triple transgenic AD model (3xTg-AD). This is an increasingly used model of AD, which overexpresses APPSwe and tauP301L, as well as carries a PS1M146V knock-in mutation, and is currently the only existing transgenic model with both $\mathrm{A} \beta$ and tau neuropathology. ${ }^{8}$ An accumulation of intraneuronal $A \beta$ occurs early, which decreases as extracellular plaques are deposited, similar to the development of disease and in brains of patients with Down's syndrome. ${ }^{15}$

Previous transgenic studies ${ }^{3,4}$ have shown that in the double AD APP/PS1 mouse line, the level of RGC apoptosis increased with age, being more preponderant at 27 than at 7.8 months. However, in these studies aged (27-months old) wildtype congeners were not assessed. Figure 6a demonstrates the typical appearance of the eye of a 14-month-old 3xTg-AD model imaged at $2 \mathrm{~h}$ after intravitreal PI and annexin-IR treatment alongside the similarly imaged eye of an 18-monthold control (PS1KI transgenic mouse that shows no $\mathrm{A} \beta$ and tau neuropathology ${ }^{16}$ ) (Figure 6b). To quantify the levels of the different phases of cell death, we again used data generated from co-localisation scatter plot analysis, which showed significantly more RGCs in the phase of early apoptosis $(P<0.05$, green, $A n x+)$ and less necrosis $(P<0.05$, red, $\mathrm{PI}+)$ in 3xTg-AD compared with aged control (Figure $6 \mathrm{c}$ ). These data show that, although a low level of apoptotic and necrotic cell death occurs as a correlate of normal healthy ageing in mice, in $A D$ mice, there is a significant increase in the relative numbers of RGCs in early-phase apoptosis.

We next investigated whether oxidative stress in AD eyes influenced the phase of cell death and whether this could be visualised in vivo. As intraneuronal $\mathrm{A} \beta$ accumulation and extracellular $\mathrm{A} \beta$ plaques are the characteristics of this model, ${ }^{15}$ we hypothesised that the addition of PMA would reveal whether the level of $A \beta$ in this transgenic mouse is sufficient to modulate the retinal response in vivo. ${ }^{14}$ We therefore compared the effects of PMA in 14-month-old 3xTg-AD animals (Figure 6d) and control aged animals (Figure 6e). PMA seemed to increase the level of annexin staining in the treated eyes of both animals. However, quantification revealed that although PMA treatment significantly increased early-phase apoptosis in both control and 3xTg-AD eyes $(P<0.05$, Figures $6 f$ and $g)$, the magnitude of the increase was far less in control eyes than in 3xTg-AD (30.3\% in aged control, $61.4 \%$ in $3 \times T$ Tg-AD). PMA also decreased late apoptosis $(P<0.05)$ in the 3xTg-AD model and necrosis $(P<0.05)$ in control eyes.

These findings show that PMA stimulates a higher rate of early-phase apoptosis (annexin V staining alone) in 3xTg-AD animals, and are consistent with the data in Figure 4 showing a similar effect of PMA in the model of acute RGC death mediated by $\mathrm{A} \beta$.

Figure 4 Analysis of cell death phases in a mouse model of $A \beta$-induced RGC death. (a, b) Wide-angle retinal images showing the $A \beta$-treated right eye (a) and $\mathrm{A} \beta+$ MK801-treated left eye (b) of a single mouse. MK801 reduces the number of annexin V (green)- and PI (red)-positive cells $72 \mathrm{~h}$ after intravitreal $\mathrm{A} \beta(25-35)$ application. To assess the magnitude of late apoptosis, we studied the co-localisation of $\mathrm{PI}$ and annexin V using ImageJ software with the Intensity Correlation Analysis plugin. ${ }^{12}$ (c, d) Scatter plots show the distribution of annexin and PI fluorescence intensity in the eyes shown in (a, b), A $\beta$ alone (c) and A $\beta+$ MK801 (d). (e) Thresholds were set automatically by the plugin for each analysis (colour overlay in (c, d)), and quantification of each cell death phase was performed from generated data (e). MK801 treatment reduced the overall levels of cell death (blue, total), mostly by reducing the number of cells in late-phase apoptosis (yellow, Anx $+/ \mathrm{PI}+$ ) and necrosis (red, $\mathrm{PI}+$ ). (f, $\mathbf{g})$ Wideangle retinal images showing the $\mathrm{A} \beta$-treated right eye ( $\mathrm{f}$ ) and $\mathrm{A} \beta+\mathrm{PMA}$-treated left eye $(\mathrm{g})$ of a single mouse. (h, i) PMA increased the level of annexin $\mathrm{V}$ (green) and PI (red) positivity, as shown in the corresponding scatter plots. (j) Quantification of all treated animals shows that PMA significantly $(P<0.05)$ increases the overall levels of cell death (blue, total), most of this being due to a significant $(P<0.05)$ increase in the number of cells in the early phase of apoptosis (green, Anx + ). (k) Comparison of cell phases in all $\mathrm{A} \beta$ and SSP models using co-localisation scatter plot analysis. At the previously established time points of maximal apoptosis ( $\mathrm{A} \beta=72 \mathrm{~h}$ and SSP $=2 \mathrm{~h}$ ), both models show a similar trend, with no significant difference in the magnitude of any phase of cell death. However, both models show significantly more RGCs in the phases of late apoptosis $(P>0.05$, yellow, $A n x+/ P I+)$ and necrosis $(P>0.05$, red, $P I+)$ than early apoptosis (green, Anx +$)$. Error bars $95 \%$ confidence intervals, ${ }^{*} P<0.05$ 

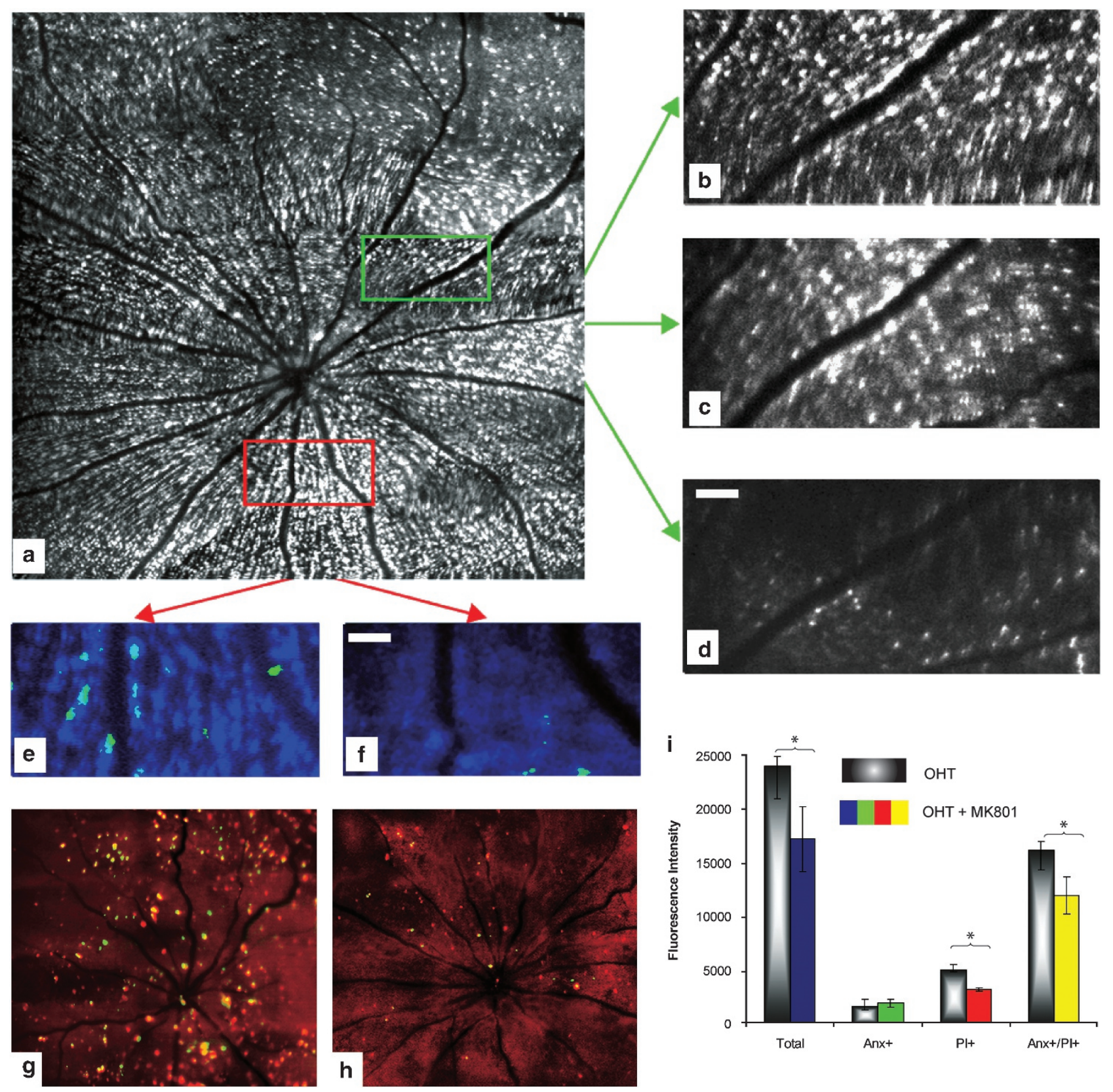

Figure 5 Natural history of cell death in a chronic ocular hypertensive (OHT) model of neurodegeneration. (a-f) Cells were retrogradely labelled using Dil (a), and analysed at intervals over 8 weeks. The higher magnification image (green box in (a)) taken at time zero (b) shows the normal density of RGCs, with little change at 3 weeks (c). However, there was marked RGC dropout 8 weeks after surgical induction of elevated IOP (d). When the data were analysed with respect to the identification of apoptosis in these same cells, it was clear that those RGCs that were annexin V positive at 3 weeks (e) were no longer identifiable at 8 weeks (f), confirming that cells originally classified as undergoing apoptosis degenerated 5 weeks later. (g) Only a small proportion of RGCs was identified in early-phase apoptosis (annexin V only) compared with late-phase apoptosis (both annexin V and PI staining) at 3 weeks. (h) MK801 treatment of animals at the time of surgery significantly reduced the number of both apoptotic and necrotic cells 3 weeks after treatment. (i) Quantitative analysis of the co-localisation data for $\mathrm{Pl}$ and annexin $\mathrm{V}$ confirmed that significantly more cells were in late-phase apoptosis $(P>0.05$, yellow, Anx $+/ P I+)$ than in necrosis (red, $\mathrm{PI}+)$. Treatment with MK801 led to a significant reduction $(P<0.05)$ in the number of dying cells (total), mostly because of a significant decrease in the levels of necrosis $(P>0.05$, red, $\mathrm{PI}+)$ and late apoptotic $(P>0.05$, yellow, Anx $+/ \mathrm{PI}+)$ cell death. Scale bar $100 \mu \mathrm{m}$ as indicated, error bars represent $95 \%$ confidence intervals, ${ }^{*} P<0.05$

\section{Discussion}

Neuronal cell loss is the key functional event in all neurodegenerative disorders, with apoptosis and necrosis being central to both acute and chronic degenerative processes. ${ }^{17}$ We report here, for the first time in vivo, a technique that enables temporal resolution and quantification of the early and late phases of apoptosis and necrosis of single nerve cells using different models of disease.

The methodology permits the tracking of changes in the same cell in the same eye over hours, days, weeks and months in acute, chronic and treated glaucoma and Alzheimer 

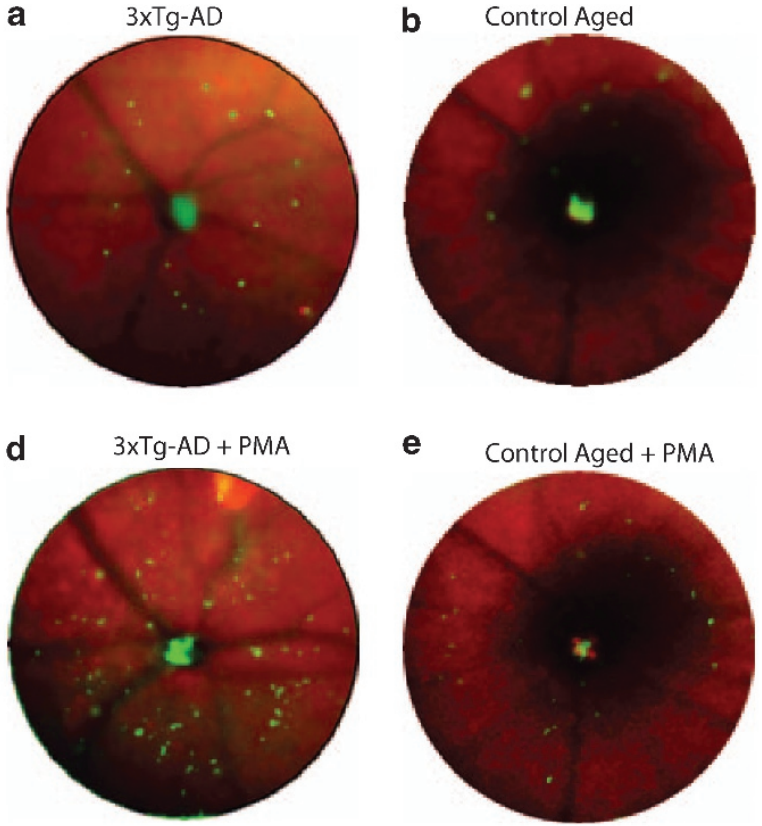

C
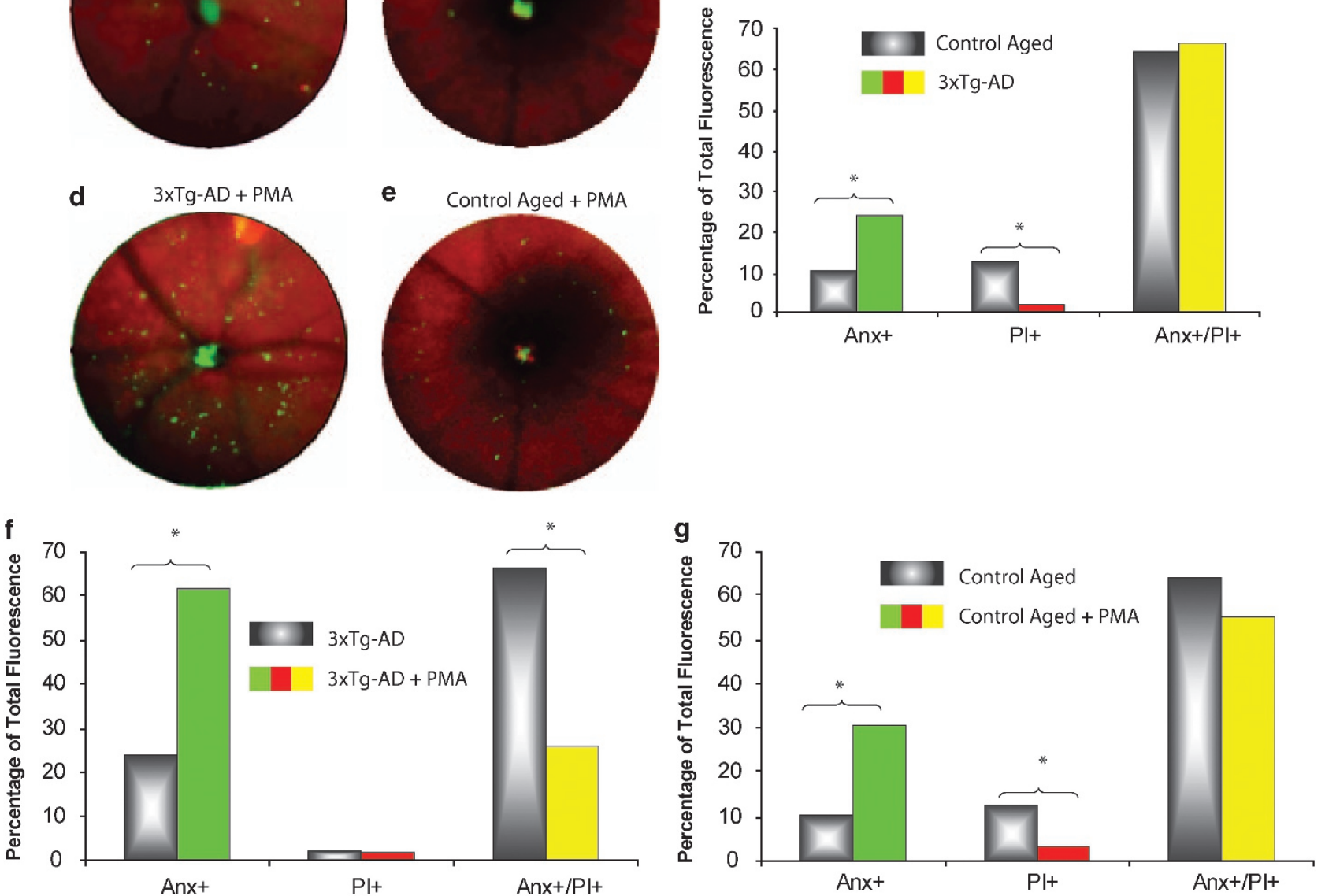

Figure 6 Retinal cell death in the Alzheimer Triple Transgenic model. (a, b) Representative wide-angle retinal images of a 14-month-old Alzheimer Triple Transgenic (3xTg-AD, a) mouse acquired $2 \mathrm{~h}$ after intravitreal PI (red) and annexin (green), compared with (b) an 18-month-old PS1KI control mouse. (c) Quantitation of co-localisation scatter plot data showing significantly more RGCs in the early phase of apoptosis $(P<0.05$, green, Anx +$)$ and relatively fewer necrotic cells $(P<0.05$, red, $P I+)$ in the 3xTgAD model compared with control. (d, e) Retinal images of the eyes of a 14-month-old 3xTg-AD animal (d) and a control aged animal (e) acquired $2 \mathrm{~h}$ after PMA was administered at the same time as intravitreal annexin (green) and PI (red). (f, g) Quantification of the data generated by co-localisation scatter plot analysis showed that PMA treatment significantly increased early apoptosis $(P<0.05$, green, Anx +$)$ and decreased late apoptosis $(P<0.05$, yellow, Anx $+/ P I+)$ in the 3xTg-AD model $(g)$. PMA also increased the level of early apoptosis in aged control animals $(P<0.05$, green, $\mathrm{Anx}+)$, although not to the same extent ( $30.3 \%$ in aged control as opposed to $61.4 \%$ in $3 x \mathrm{Tg}$ AD). ${ }^{*} P<0.05$

models of neurodegeneration. It has enabled us to identify a numerical preponderance of late-phase versus early-phase apoptotic cells in vivo, and reinforced the commonalities between disease mechanisms in the different models studied. The predominance of late-phase apoptosis may reflect not only the relatively short duration of the early phase compared with the late phase (previously estimated as within 1 hour ex vivo $^{18}$ and suggesting that the relative likelihood of detecting cells in early apoptosis is low at any given time ${ }^{19}$ ) but also, as we show here ('similar profiles' in Figure 3), the coincidental appearance of annexin $\mathrm{V}$ and $\mathrm{PI}$ positivity in many apoptotic cells.

The shared characteristics of cell death patterns in different disease models is an important aspect of the technique presented here, with particular relevance to the design of more effective and targeted therapeutics. NMDA antagonists have long been advocated in neuroprotective strategies ${ }^{20,21}$ in both $\mathrm{AD}^{22}$ and glaucoma, ${ }^{9,21}$ and MK801 effectively inhibited both apoptosis and necrosis in our different models, consistent with the role of $\mathrm{Ca}^{2+}$ in mediating both forms of cell death. However, these findings support the use of our technique to investigate more specific anti-apoptotic and anti-necrotic strategies with well-defined targets, with potentially greater clinical application. It may be that the use of these strategies will depend on the stage of the disease in the patient, which may eventually be defined by an apoptotic/ necrotic index, a ratio that may indicate disease severity and progression.

A major advantage of our approach is that it allows a temporal delineation of the natural history of neuronal loss. Various attempts to discriminate between the various modes of cell death in neurodegenerative diseases such as AD have 
been inconclusive, with mixed features of apoptosis, necrosis and autophagy being detected through a variety of histological and biochemical strategies, which are difficult to extrapolate to the dynamic events that occur in vivo. ${ }^{23}$ The overlap between apoptosis and necrosis pathways has been linked to insult exposure, ${ }^{24}$ with necrosis being associated with excessive pathological neuronal loss, ${ }^{25}$ now considered to be the 'default' cell death pathway when apoptotic cell death is inhibited. ${ }^{26}$ Further investigation to elucidate whether the mode of neurodegenerative cell death is directly determined by the strength of the cell death stimulus is needed.

On the whole, most glaucoma studies have focused on the development of generic RGC loss rather than on the specific mode of cell death, although some have proposed apoptosis as the predominant pathway. ${ }^{27-29}$ Our results lead us to suggest that, although apoptosis may be involved in the primary phase of degeneration in animal models, necrosis is probably the major cell death pathway in secondary degeneration ${ }^{30}$ in glaucoma. It will be interesting to use the imaging technique developed in this study, in combination with the many fluorescent cell-permeant probes available for caspase activation, $\mathrm{Ca}^{2+}$ fluxes, mitochondrial function and so on, to examine degeneration phases in other transgenic neurodegeneration models, as the balance between apoptosis and necrosis is still unclear in chronic disease. Furthermore, delineation of the timing of such events and their association with RGC apoptosis will highlight processes that precede the irreversible stages of cellular degeneration, and provide improved and specific targets for neuroprotection.

Annexin 5 is already used systemically and safely in patients, ${ }^{31,32}$ and there are a number of necrosis markers that are already being used in clinical trials, such as $99 \mathrm{mTc}$ pyrophosphate, $111 \mathrm{In}$-antimyosin and $99 \mathrm{mTc}$-glucarate. ${ }^{32}$ Hence, although it is unlikely that PI can be used in patients, there are alternative agents that are potentially available to allow this methodology to be translated directly to the clinic. The ability to differentiate between apoptosis and necrosis and non-invasively track cell death in neurodegenerative pathologies could have an important role in medical diagnostics and therapy management. Furthermore, we believe that the concept of an early apoptotis/necrosis ratio with a correlation to disease activity may provide an invaluable tool for staging neurodegeneration. There is increasing evidence of the potential reversibility of early apoptosis, suggesting that a high early apoptosis/necrosis ratio may offer a good prognosis for treatment. ${ }^{33,34}$

In conclusion, the application of this new technology has far-reaching implications for studying intracellular processes at the single-cell level in vivo. Although the equipment we use in these studies has been customised to suit animal models, the instruments are essentially the same as those used in hospitals and clinics around the world. This raises the possibility that in the near future, clinicians may be able to assess retinal nerve cell death in vivo as a method of monitoring disease progression and treatment efficacy. Whether a single snapshot or long-term in vivo observation over many weeks is needed, the ability to visualise and track changes in cell viability offers the potential for major advances in the diagnosis and management of neurodegenerative diseases.

\section{Materials and Methods}

Animal models. For our investigations in rats, we used established models of retinal neuronal apoptosis. ${ }^{1}$ All procedures complied with local and national regulations and were performed under general anaesthesia. Adult dark Agouti (DA) rats, $150-200 \mathrm{~g}$, were used in all rat experiments.

For the experimental glaucoma studies, we used an established model of ocular hypertension. Eighteen rats were operated to elevate their IOP by injection of hypertonic saline solution $(1.8 \mathrm{M})$ into episcleral veins as previously described. ${ }^{1}$ Contralateral, unoperated eyes were used as controls. Animals were imaged at 0,3 , 8 and 16 weeks, with at least three animals at each time point being killed for histological analysis immediately after imaging. A subgroup of animals $(n=4)$ was treated at the time of surgery with the NMDA antagonist MK801 $(0.6 \mathrm{nmol}$; Tocris Cookson, Bristol, UK). These animals were imaged at 3 weeks and then killed for histology.

For analysis of SSP-induced RGC apoptosis, 14 DA rats were given $0.5 \mu \mathrm{g}$ of intravitreal SSP in $5 \mu$ l phosphate-buffered saline (PBS), both from Sigma-Aldrich (Dorset, UK), to induce RGC apoptosis as previously described. ${ }^{1}$ Animals were imaged immediately for up to $6 \mathrm{~h}$, after which they were killed for histology.

For the analysis of $A \beta$, freshly prepared $A \beta_{25-35}$ (Sigma-Aldrich, $\left.3.40 \mathrm{nmol}\right)^{35}$ was intravitreally injected into C57 mice $(n=10){ }^{11}$ The animals were imaged at $72 \mathrm{~h}$. For AD 3xTg-AD studies, four eyes (all female, 14 months), with two eyes from aged control mice (PS1Kl; female, 18 months), were assessed. 3xTg-AD and control PS1KI mice were obtained from a colony (maintained at CeSI) established after they were received as a generous gift from Frank Laferla. Intravitreal PMA $(1 \mu \mathrm{l}$ of $10 \mu \mathrm{g} / \mathrm{ml}$ ) was administered to the left eyes of $3 \times \mathrm{Tg}-\mathrm{AD}$ animals, at the same time as annexin $\mathrm{V}$ and $\mathrm{PI}$.

Fluorescent in vivo labelling. To identify RGC, retinas were retrogradely labelled using different agents in pilot studies by their application to both superior colliculi, as previously described. ${ }^{1}$ Pilot studies identified Dil (10\% solution; Molecular Probes, Cambridge Biosciences, Cambridge, UK) as being better than DiAsp (4-Di-10-Asp, Fluorogold or Rhodamine B). Dil was applied 10 days before all the rat treatment procedures detailed above. Dil is a long-chain dialkylcarbocyanine lipophilic neuronal tracer, which does not affect cell viability, development or the basic cellular physiological properties. ${ }^{36}$

Using a method that we recently described, rats were administered $2.5 \mu \mathrm{g}$ of intravitreal Alexa Fluor 488-labelled annexin V in $5 \mu \mathrm{l}$ PBS for identification of RGC apoptosis, $2 \mathrm{~h}$ before in vivo imaging. ${ }^{1}$ For mice, we used $1 \mu \mathrm{l}$ of a $0.25 \mathrm{mg} / \mathrm{ml}$ solution of IR-labelled annexin (Annexin-IR) through intravitreal injections as previously described. ${ }^{37}$

To identify necrotic RGCs in rats $(2 \mu \mathrm{l})$ and mice $(1 \mu \mathrm{l}), \mathrm{PI}(0.4 \mathrm{mg} / \mathrm{ml}, 1 \%$ solution, Sigma-Aldrich) was administered intravitreally at the same time as annexin, $2 \mathrm{~h}$ before in vivo imaging. PI intercalates into nucleic acids, binding to double-stranded DNA, but only crosses the plasma membrane of non-viable cells. Cells that have lost membrane integrity are thus identified by fluorescent red PI staining throughout the nuclei.

Imaging. All rats had in vivo retinal imaging performed at regular and set intervals after experimental treatment using a prototype Zeiss CSLO, as previously described. ${ }^{1,11}$ Imaging was performed under general anaesthesia with animals being held in a stereotaxic frame, with their pupils dilated. Different lasers and filters were used for different fluorochromes: for excitation of Alexa Fluor 488, an argon laser wavelength of $488 \mathrm{~nm}$ and a solid state photodetector with a $521 \mathrm{~nm}$ cut-off filter and a $550 \mathrm{~nm}$ cut-on filter were used; for Dil, a diode-pumped crystal green laser with a wavelength of $532 \mathrm{~nm}$ was used with $550 \mathrm{~nm}$ cut-off and $600 \mathrm{~nm}$ cut-on filters; the same laser was used for PI visualisation, but with a $650 \mathrm{~nm}$ cut-off. The cSLO used in this study is the same as that described previously, with modifications complying with the established standards for safety and light exposure. ${ }^{1,37}$ We have developed a purpose-written software to allow optimal processing of low-light fluorescent signals, which has greatly aided our live cell imaging. ${ }^{1}$ Monochrome images from each area were acquired using the appropriate laser setup and colourised. Three-colour images were produced by combining each of the grey scale images from the cSLO into the red (PI), green (annexin 488) and blue channels (Dil) of an RGB colour image. Images were processed in this way because red, green and blue (RGB) colours are universally accepted as the most informative methods for showing co-localisation. The ImageJ Intensity Correlation Analysis plugin was applied to generate co-localisation scatter plots and colour thresholds. ${ }^{12}$ Colour and raw scatter plots of annexin and PI fluorescence intensity were constructed; hence, the data could be analysed in a similar manner to that of the 
data derived by in vitro FACS analysis. Separation of the different phases of cell death according to whether they were annexin $V$ positive (early apoptosis), annexin $\mathrm{V}$ and PI double positive (late apoptosis) or PI positive (necrosis) was enabled by the adoption of thresholds set automatically by the plugin for each analysis Fluorescence intensity counts derived from raw scatter plots were used to quantify each phase of cell death.

In mice, we used instrumentation as previously described, ${ }^{11,37}$ with a modified cSLO (Heidelberg Retina Angiograph 2, Heidelberg Engineering, Dossenheim, Germany) and a wide-field lens $\left(55^{\circ}\right)$. The fluorescein angiogram ( $488 \mathrm{~nm}$ argon laser) and indocyanine green (IR $790 \mathrm{~nm}$ diode laser) settings were used to detect $\mathrm{PI}$ - and IR-labelled annexin, respectively. The same software was used to analyse video-sequenced images as described before, with whole retinal monochrome images of each eye recorded and colourised. Dual-color images were produced by combining each of the grey scale images from the cSLO into the red $(\mathrm{PI})$ and green (IR annexin) channels of an RGB colour image.

Analysis. ImageJ software was used with specific plugins to create 3D surface plots, RGB projections and to analyse co-localisation ( $\mathrm{JaCOP}$ and Intensity Correlation Analysis). Graphical data for mean values were contrived with $95 \%$ confidence intervals, and comparisons across groups were performed using ANOVA or Student's t-test for paired eyes, with $P<0.05$ as the significance level.

\section{Conflict of interest}

Professors M Francesca Cordeiro, Frederick W Fitzke and Stephen E Moss are named inventors on a patent application covering the technology disclosed in this report.

Acknowledgements. We acknowledge support from The Wellcome Trust, The Foundation Fighting Blindness, Dr. Annelie Maass and Mr. Vy Luong.

1. Cordeiro MF, Guo L, Luong V, Harding G, Wang W, Jones HE et al. Real-time imaging of single nerve cell apoptosis in retinal neurodegeneration. Proc Natl Acad Sci USA 2004 101: 13352-13356.

2. Kvam E, Nannenga BL, Wang MS, Jia Z, Sierks MR, Messer A. Conformational targeting of fibrillar polyglutamine proteins in live cells escalates aggregation and cytotoxicity. PloS One 2009; 4: e5727.

3. Ning A, Cui JZ, To E, Hsiao Ashe K, Matsubara JA. Amyloid beta deposits lead to retinal degeneration in a mouse model of Alzheimer disease. Invest Ophthalmol Vis Sci 2008; 49 : 5136-5143.

4. Shimazawa M, Inokuchi Y, Okuno T, Nakajima Y, Sakaguchi G, Kato A et al. Reduced retinal function in amyloid precursor protein-over-expressing transgenic mice via attenuating glutamate-N-methyl-d-aspartate receptor signaling. J Neurochem 2008; 107 279-290.

5. Guo L, Duggan J, Cordeiro MF. Alzheimer's disease and retinal neurodegeneration Curr Alzheimer Res 2009 [e-pub ahead of print 1 December 2009].

6. Guo L, Salt TE, Luong V, Wood N, Cheung W, Maass A et al. Targeting amyloid-beta in glaucoma treatment. Proc Natl Acad Sci USA 2007; 104: 13444-13449.

7. Huerta S, Goulet EJ, Huerta-Yepez S, Livingston EH. Screening and detection of apoptosis. J Surg Res 2007; 139: 143-156.

8. Sensi SL, Rapposelli IG, Frazzini V, Mascetra N. Altered oxidant-mediated intraneuronal zinc mobilization in a triple transgenic mouse model of Alzheimer's disease. Exp Gerontol 2008; 43: 488-492.

9. Guo L, Salt TE, Maass A, Luong V, Moss SE, Fitzke FW et al. Assessment of neuroprotective effects of glutamate modulation on glaucoma-related retinal ganglion cell apoptosis in vivo. Invest Ophthalmol Vis Sci 2006; 47: 626-633.

10. Belmokhtar CA, Hillion J, Segal-Bendirdjian E. Staurosporine induces apoptosis through both caspase-dependent and caspase-independent mechanisms. Oncogene 2001; 20: 3354-3362.

11. Maass A, von Leithner PL, Luong V, Guo L, Salt TE, Fitzke FW et al. Assessment of rat and mouse RGC apoptosis imaging in vivo with different scanning laser ophthalmoscopes. Curr Eye Res 2007; 32: 851-861.

12. Li Q, Lau A, Morris TJ, Guo L, Fordyce CB, Stanley EF. A syntaxin 1, Galpha(o), and $\mathrm{N}$-type calcium channel complex at a presynaptic nerve terminal: analysis by quantitative immunocolocalization. J Neurosci 2004; 24: 4070-4081.

13. Miguel-Hidalgo JJ, Alvarez XA, Cacabelos R, Quack G. Neuroprotection by memantine against neurodegeneration induced by beta-amyloid(1-40). Brain Res 2002; 958: 210-221.

14. Abramov AY, Duchen MR. The role of an astrocytic NADPH oxidase in the neurotoxicity of amyloid beta peptides. Philos Trans R Soc Lond B Biol Sci 2005; 360: 2309-2314.

15. LaFerla FM, Green KN, Oddo S. Intracellular amyloid-beta in Alzheimer's disease. Nat Rev Neurosci 2007; 8: 499-509.

16. Oddo S, Caccamo A, Shepherd JD, Murphy MP, Golde TE, Kayed R et al. Tripletransgenic model of Alzheimer's disease with plaques and tangles: intracellular Abeta and synaptic dysfunction. Neuron 2003; 39: 409-421.

17. Vila M, Przedborski S. Targeting programmed cell death in neurodegenerative diseases. Nat Rev Neurosci 2003; 4: 365-375.

18. Cellerino A, Galli-Resta L, Colombaioni L. The dynamics of neuronal death: a time-lapse study in the retina. J Neurosci 2000; 20: RC92.

19. Lecoeur H, Chauvier D, Langonne A, Rebouillat D, Brugg B, Mariani J et al. Dynamic analysis of apoptosis in primary cortical neurons by fixed- and real-time cytofluorometry. Apoptosis 2004; 9: 157-169.

20. Lipton SA. Paradigm shift in neuroprotection by NMDA receptor blockade: memantine and beyond. Nat Rev 2006; 5: 160-170.

21. Cheung ZH, So KF, Lu Q, Yip HK, Wu W, Shan JJ et al. Enhanced survival and regeneration of axotomized retinal ganglion cells by a mixture of herbal extracts. J Neurotrauma 2002; 19: 369-378.

22. Farlow MR. NMDA receptor antagonists. A new therapeutic approach for Alzheimer's disease. Geriatrics 2004; 59: 22-27.

23. Andorfer C, Acker CM, Kress Y, Hof PR, Duff K, Davies P. Cell-cycle reentry and cell death in transgenic mice expressing nonmutant human tau isoforms. J Neurosci 2005; 25 : 5446-5454.

24. Zeiss CJ. The apoptosis-necrosis continuum: insights from genetically altered mice. Vet Pathol 2003; 40: 481-495.

25. Krysko DV, Vanden Berghe T, D'Herde K, Vandenabeele P. Apoptosis and necrosis: detection, discrimination and phagocytosis. Methods (San Diego, CA) 2008; 44: 205-221.

26. Golstein P, Kroemer G. Cell death by necrosis: towards a molecular definition. Trends Biochem Sci 2007; 32: 37-43.

27. Garcia-Valenzuela E, Shareef S, Walsh J, Sharma SC. Programmed cell death of retinal ganglion cells during experimental glaucoma. Exp Eye Res 1995; 61: 33-44.

28. Harwerth R, Carter-Dawson L, Shen F, Smith E, Crawford M. Ganglion cell losses underlying visual field defects from experimental glaucoma. Invest Ophthalmol Vis Sci 1999; 40: 2242-2250

29. Kerrigan-Baumrind L, Quigley H, Pease M, Kerrigan D, Mitchell R. Number of ganglion cells in glaucoma eyes compared with threshold visual field tests in the same persons. Invest Ophthalmol Vis Sci 2000; 41: 741-748.

30. Moalem G, Yoles E, Leibowitz-Amit R, Muller-Gilor S, Mor F, Cohen IR et al. Autoimmune $T$ cells retard the loss of function in injured rat optic nerves. J Neuroimmunol 2000; 106: 189-197.

31. Reutelingsperger $\mathrm{CP}$, Dumont $\mathrm{E}$, Thimister PW, van Genderen $\mathrm{H}$, Kenis $\mathrm{H}$, van de Eijnde $\mathrm{S}$ et al. Visualization of cell death in vivo with the annexin A5 imaging protocol. J Immunol Methods 2002; 265: 123-132.

32. De Saint-Hubert M, Prinsen K, Mortelmans L, Verbruggen A, Mottaghy FM. Molecular imaging of cell death. Methods (San Diego, CA) 2009; 48: 178-187.

33. Tang HL, Yuen KL, Tang HM, Fung MC. Reversibility of apoptosis in cancer cells. Br J Cancer 2009; 100: 118-122.

34. Masri C, Chandrashekhar Y. Apoptosis: a potentially reversible, meta-stable state of the heart. Heart Fail Rev 2008; 13: 175-179.

35. Dahlgren KN, Manelli AM, Stine Jr WB, Baker LK, Krafft GA, LaDu MJ. Oligomeric and fibrillar species of amyloid-beta peptides differentially affect neuronal viability. J Biol Chem 2002; 277: 32046-32053

36. Honig MG, Hume RI. Dil and diO: versatile fluorescent dyes for neuronal labelling and pathway tracing. Trends Neurosci 1989; 12: 333-335, 340, 341.

37. Schmitz-Valckenberg S, Guo L, Maass A, Cheung W, Vugler A, Moss SE et al. Real-time in-vivo imaging of retinal cell apoptosis after laser exposure. Invest Ophthalmol Vis Sci 2008; 49: 2773-2780.

(c)

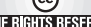
licensed under a Creative Commons Attribution-Noncommercial-No Derivative Works 3.0 License. To view a copy of this License, visit http:// creativecommons.org/licenses/by-nc-nd/3.0/

Supplementary Information accompanies the paper on Cell Death and Disease website (http://www.nature.com/cddis) 